\title{
A Survey on Asynchronous MAC protocols in Wireless Sensor Networks
}

\author{
Arvind Kakria \\ PEC University of Technology, \\ Sector-12, \\ Chandigarh, India
}

\author{
Trilok Chand Aseri, Ph.D. \\ PEC University of Technology, \\ Sector-12, \\ Chandigarh, India
}

\begin{abstract}
Wireless Sensor network (WSN) plays an important role in applications like surveillance, agriculture monitoring, health monitoring, industrial automation etc. Large number of low cost sensor nodes are distributed that can communicate with each other. WSN MAC protocols can be classified in to Synchronous, Asynchronous, Multiple channels and Frame based [2].Our paper focuses on the latest progresses in Asynchronous MAC protocols
\end{abstract}

\section{Keywords}

Delivery, Performance, Overhead, Synchronous, Asynchronous

\section{INTRODUCTION}

Wireless sensor network (WSN)[1] has played an important role in the area of environment, agriculture monitoring ,traffic control , disaster management etc. Large number of low cost sensor nodes is distributed in specific area of interest such that nodes can communicate with each other. WSN networks have two classifications: Structured and Unstructured. In structured WSN the position of placing the nodes are predetermined so that perfect coverage is provided. In Unstructured WSN the nodes are placed in Ad-hoc manner. Sensor nodes are operated by small battery that consumes lot of energy during transmission of signal so energy efficiency is one of the main criterion for designing any MAC protocol [2].Radio is the main component of any sensor node and consumes lot of power during transmission and listening. Therefore efficient MAC protocol can increase life time of any sensor network. MAC layer protocols in Wireless sensor network can be divided in to four categories depending upon how they access the channel: Contemporaneous or Synchronous awaking of nodes, Asynchronous or noncontemporaneous awaking of nodes, Slotting of frames and approach based on multi channel [2]. Duty cycle is the main feature of Synchronous and Asynchronous protocols in which any node can alternate between active and sleep states to save energy. Nodes can only communicate when they are in active state. In Synchronous MAC protocols all nodes wake up at same time whereas Asynchronous MAC protocols are based on the communication between two nodes having different sleep/active schedules. This paper includes the survey of Asynchronous MAC protocols and how communication is made among various nodes with different sleep/awake schedules. Various factors that cause the energy consumption are summarized as follows:
1. Listening idly by nodes: Lot of energy is wasted when a node listen idly to the channel. It happens because node is unaware of the fact that neighboring node has some data to send or not. This consumes energy and is one of the main reasons of energy wastage.

2. Collision of packets: when two packets are transmitted collisions can occur. So the data has to be retransmitted again and this process consumes energy.

3. Overhearing by Nodes: Overhearing is that when a node receives a packet that is not meant for it. It also consumes energy and needs to be minimized.

4. Overhead by packets: Control packets play an important role in sending, transmitting and receiving data. But due to this overhead is there in communication and contributes to wastage of energy.

\section{ASYNCHRONOUS/ CONTEMPORANEOUS MAC PROTOCOLS}

\subsection{Preamble Sampling based on CSMA}

In CSMA-Preamble Sampling (CSMA-PS) [2][3], active and sleep modes are used in which nodes alternates between active and sleep mode. Here node periodically wakes up to sample the channel. This is done to minimize active duration of the nodes and to increase the sleep interval of nodes. In sampling a node detects that whether a channel is idle or not. If a channel is idle it goes back to sleep and if any activity is detected on the channel it keeps in active state. According to the length of the channel sampling interval a long preamble is used.

\subsection{Protocols based on Sparse Technology}

STEM (Sparse Topology and Energy Management)[4][5],has two phases : transfer phase and wake up phase. The two phases are separated through use of two separate radios. The initiator sends stream of beacon in band $\mathrm{f} 1$ which contains address of target and initiator node. On receiving the beacon target node sends acknowledgement in band $\mathrm{f} 2$. This reduces latency in set up. Figure 1.represents the timing diagram of STEM. Here active time of receiver can be reduced but the energy of wake up signal is very high. 


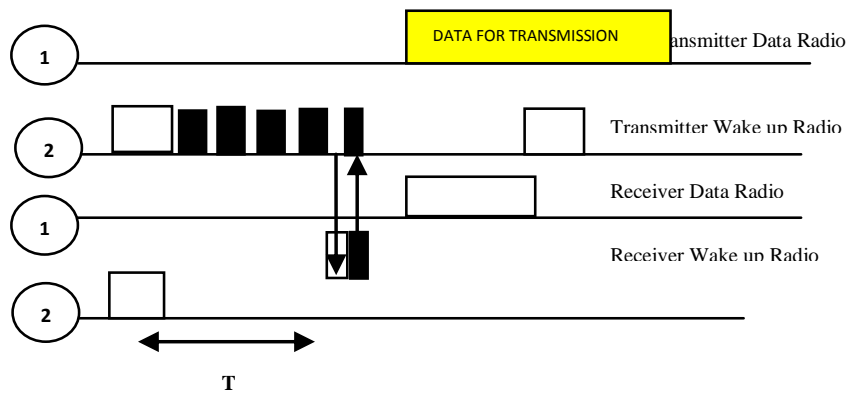

Fig 1.Timing diagram of STEM

\subsection{Wise-MAC}

Wise-MAC [6] is a protocol which reduces the overhead at the transmitter and receiver. Wise-MAC minimizes the wakeup preamble by acquiring the knowledge of sampling schedule of its direct neighbors. Using preamble of minimum size transmission gets started. Here sampling information is contained in acknowledge packet. Here no set-up signaling and network-wide time synchronization is necessary. Non persistence carrier sensing is used with back off time and a medium reservation preamble of randomized duration so as to avoid collisions. Figure 2 shows the timing diagram of the protocol after the arrival of an event (i.e. packet to be forwarded) at the source. The source waits for the sampling schedule and sends a preamble just at the right time with a wake-up preamble of minimum size ( $\mathrm{T}$ preamble) to make sure to hit the destinations receiver window if the sampling schedule and the time of the last synchronization is known. Disadvantage of this protocol is that the short preamble alleviates the overhearing problem and channel capacity is increased.

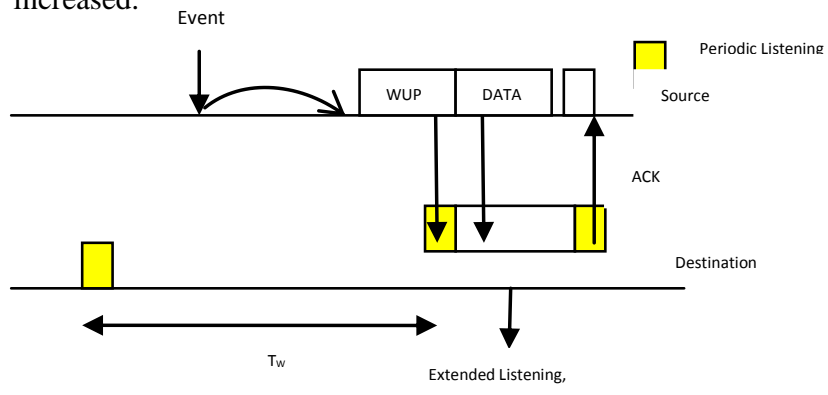

Fig 2. Timing diagram of Wise-MAC

\subsection{MAC protocol based on outlier detection}

B-MAC (Berkeley MAC) introduces an outlier detection method to improve the quality of Clear channel assessment (CCA). Immediately after transmitting a frame every node take signal strength samples at times when channel is supposed to be clear. These samples are entered into a FIFO queue. Nodes which needs to perform CCA searches for outliers in the received signal such that channel energy is significantly below the noise floor. B-MAC declares the channel is clear if an outlier is detected during the sampling of channel as a valid transmission could never have an outlier significantly below the noise floor. B-MAC declares that the channel is busy if no outliers are detected. As false positives lead to lower duty cycle B-MAC names its preamble as Low Power Listening (LPL). This protocol has an advantage of low power of operation and effective collision avoidance but also has a disadvantage that it is unable to provide hidden terminal support and no message fragmentation.

\subsection{B-MAC +}

As discussed above B-MAC+ [8] is an improvement of BMAC which has been discussed above. In B-MAC+ pattern of wake-up preamble of B-MAC is replaced with a new pattern that consists of information of the size of the remaining part of the preamble which has not been transmitted. Receivers use this information to avoid wait states during significant portions of the preamble transmission time. The wake up preamble of B-MAC is divided in to chunks to obtain the wake-up preamble of B-MAC+. Each chunk is a countdown packet, which consists of a sort preamble and a data payload. The data payload has a counter and an address. The size of remaining part of wake-up preamble is represented by counter and destination address represents the actual recipient of data packet. The main advantage of using B-MAC+ is that it avoids overhearing as preamble contains address of destination node Also B-MAC+ avoids ideal listening. The main disadvantage of B-MAC+ is that when receiver turns its radio off after getting the preamble but the sender still keeps on sending the remaining part of the preamble which causes energy wastage and introduces latency.

\subsection{Protocol based on Minimum Preamble Sample with CSMA}

To increase the efficiency of wake-up mechanism of STEM and Wise-MAC and combining the best features of both the protocols CSMA-MPS (CSMA-Minimum Preamble Sapling) [10] was developed. In STEM alternating transmit and receive slot is used during wake up to find whether the receiver's window was hit. Latest clock estimate obtained during last communication is the principle on which Wise MAC tries to hit the receiver's window but it sends out worst case preamble length based on estimate before sending transmitting data frame. CSMA-MPS uses the best features of both the protocols in which it uses the alternating transmits and receives of STEM and combines them with efficient wake-ups of Wise MAC. So there is no need to send the preamble with worst case length as alternating transmits and receive allows detecting synchronization almost immediately. To avoid ad additional request response message in separate phase wake up and data phase can be combined. To check the activity of the channel all nodes sample medium for short time with constant period T. A node that wants to contact one of its neighbors may or may not have a sampling schedule of its neighbors. If a node do not have sampling schedule of its neighbor it starts CSMA algorithm immediately, otherwise it will start its periodic listening again as the estimate of clock is available so it backs of some time.

\subsection{X-MAC}

$\mathrm{X}-\mathrm{MAC}[11]$ is a new approach to low power listening in which a short preamble is used to further reduce energy consumption and to reduce latency. Here the address information of the target is added in preamble so that non target receivers can go back to sleep as soon as possible. This addresses the problem of overhearing. Also strobed preamble is used to allow the target receiver to interrupt long preamble as it wakes up and finds that it is s target receiver. Strobed preamble is helpful in reducing the time and energy wastage which is consumed in waiting the entire preamble to complete. Here an automated algorithm is used for adapting the duty cycle of nodes to adjust the duty cycle of the nodes. Advantage of this protocol is that it reduces energy at both receiver and sender sides and also reduces latency hop. It also avoids collisions by using randomized back off and addresses the overhearing problem. This protocol also has disadvantage 
that preamble transmission still occupies the channel until the target receiver wakes up.

\subsection{Wake up Frame MAC Protocol with Wise MAC}

SyncWUF-MAC [12] is a combination of WUF and WiseMAC. The basic idea on which SyncWUF works is that sender stores the receiver's schedule. Here if $\mathrm{T}$ is small and length of WUS i.e. 4T is short and receiver schedule is received recently then short WUP is used as is used in pure WiseMAC protocol. Multiple SUFs are used in WUS to reduce unnecessary waiting time if schedule is out of date. The main advantage of using this protocol is that it is based on efficient power saving duty cycle scheduling scheme and can be implemented regardless of a particular application.

\subsection{Receiver Initiated MAC}

Receiver-initiated MAC (RI-MAC) is an asynchronous duty cycle MAC protocol that employs receiver initiated data transmission technique. For the purpose of minimizing energy depletion, RI-MAC attempts to minimize the rendezvous time between the sender and its intended receiver. The sender remains active and waits silently until the receiver explicitly signifies when to start data transmission by sending a short beacon frame. As only beacon and data transmissions occupy the medium in RI-MAC, with no preamble transmissions as in LPL (Low Power Listening) -based protocols; occupancy of the medium is significantly decreased, so that other nodes can exchange data. The receiver-initiated design in the RI-MAC not only substantially reduces overhearing, but also achieves lower collision probability and recovery cost. RI-MAC significantly improves throughput and packet delivery ratio, especially when there are contending flows such as bursty traffic or transmissions from hidden nodes. In this protocol, the nodes are scheduled to wake up periodically to verify if any data packets are intended for them. They send out a beacon, which is picked up by an awake node that has pending data packets to send. After receiving the beacon the sender node starts transmitting the data packets. On the reception of these, the receiver node sends an acknowledgement beacon (ACK).

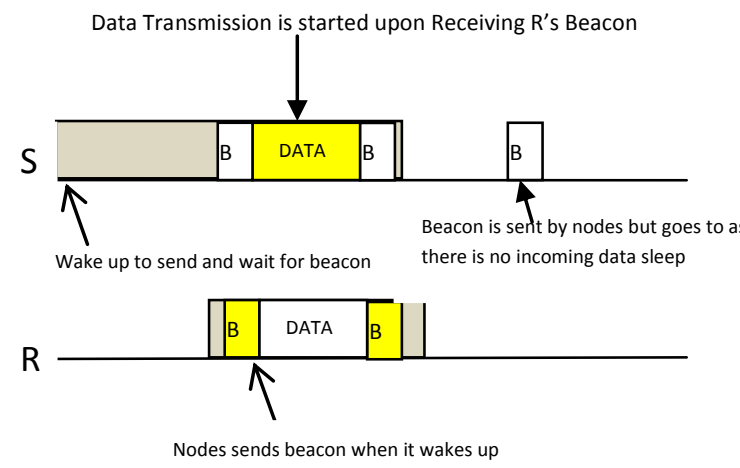

Fig 3. Timing diagram of RI-MAC

\subsection{Receiver Centric MAC Protocol}

Receiver-initiated MAC [13] (RI-MAC) is an asynchronous duty cycle MAC protocol that employs receiver initiated data transmission technique. For the purpose of minimizing energy depletion, RI-MAC attempts to minimize the rendezvous time between the sender and its intended receiver. The sender remains active and waits silently until the receiver explicitly signifies when to start data transmission by sending a short beacon frame. As only beacon and data transmissions occupy the medium in RI-MAC, with no preamble transmissions as in LPL (Low Power Listening) -based protocols; occupancy of the medium is significantly decreased, so that other nodes can exchange data. The receiver-initiated design in the RI-MAC not only substantially reduces overhearing, but also achieves lower collision probability and recovery cost. RI-MAC significantly improves throughput and packet delivery ratio, especially when there are contending flows such as bursty traffic or transmissions from hidden nodes. In this protocol, the nodes are scheduled to wake up periodically to verify if any data packets are intended for them. They send out a beacon, which is picked up by an awake node that has pending data packets to send. After receiving the beacon the sender node starts transmitting the data packets. On the reception of these, the receiver node sends an acknowledgement beacon (ACK). Figure 4 depicts the overview of RI-MAC protocol. The ACK beacon plays a dual role - first to acknowledge the reception of the data packet and second to ask for more data packets if any from the same node. RI-MAC efficiently minimizes the medium occupancy time taken by a sender and its intended receiver before they reach a common active period for data transmission. RI-MAC achieves better throughput, higher packet delivery ratio and energy efficiency than X-MAC [11]. In RI-MAC, medium access control among senders that want to transmit DATA frames to the same receiver is mainly controlled by the receiver. This design of RI-MAC makes it more efficient in detecting collisions and recovering DATA frames that are lost than B-MAC and X-MAC where the senders are hidden to each other. As a receiver expects incoming data only RI-MAC reduces overhearing within a small window after beacon transmission. With the lower cost for recovering lost DATA frames and detecting collisions, RI-MAC has higher power efficiency even when the load of network increases.

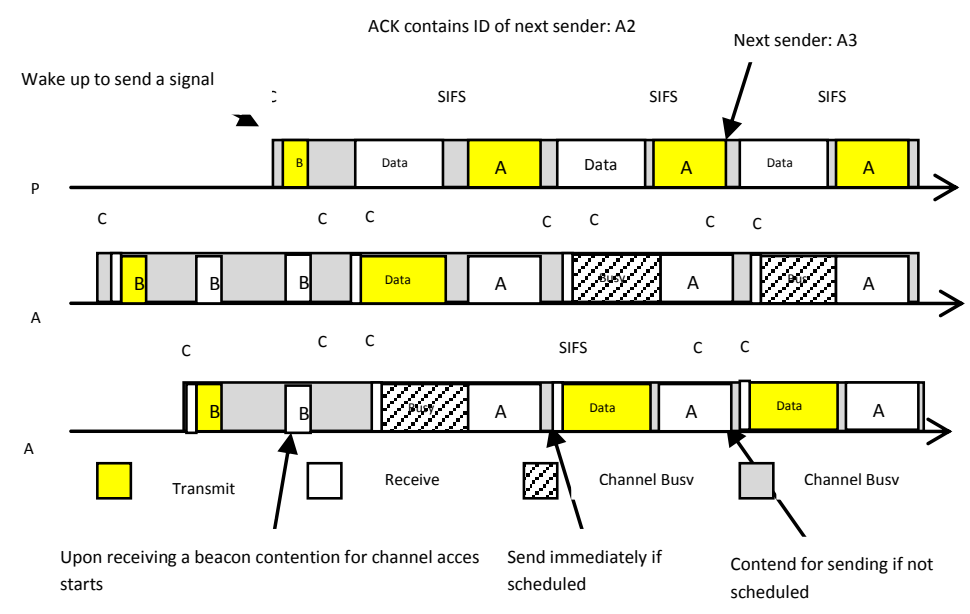

Fig 4. Timing diagram of RC-MAC

\section{REFERENCES}

[1] J. Yick, B. Mukherjeeand D. Ghosal, "Wireless sensor network survey", Computer Networks, Volume 52, Issue 12, pp. 2292-2330, August 2008.

[2] Pei Huang, Li Xiano, Soltani S, M.W Mutka, Ning Xi, "The Evolution of MAC Protocols in Wireless Sensor Networks : A Survey", Communication Survey and Tutuorials, Volume:15, Issue: 1, 2013, pp. 101-120. 
[3] J. L. Hill and D. E. Culler, "Mica: A wireless platform for deeply embedded networks," IEEE Micro, vol. 22, no. 6, pp. 12-24, November/December 2002.

[4] S. Mahlknecht and M. Bock, "CSMA-MPS: a minimum preamble sampling MAC protocol for low power wireless sensor networks," in Proc. IEEE International Workshop on Factory Communication Systems, September 2004, pp. $73-80$.

[5] C. Schurgers, V. Tsiatsis, S. Ganeriwal, and M. Srivastava, "Optimizing sensor networks in the energylatency-density design space," IEEETrans. Mobile Computing, vol. 1, no. 1, pp. 70-80, January-March 2002.

[6] A. El-Hoiydi and J.-D.Decotignie, "WiseMAC: An ultralow power MAC protocol for multi-hop wireless sensor networks," in Proc. 9 ${ }^{\text {th }}$ International Symposium on Computers and Communications (ISCC), vol. 1, JuneJuly 2004, pp. 244-251.

[7] J. Polastre, J. Hill, and D. Culler, "Versatile low power media access for wireless sensor networks," in Proc. SenSys, 2004.

[8] M. Awenuti, P. Corsini, P. Masci, and A. Vecchio, "Increasing the efficiency of preamble sampling protocols for wireless sensor networks," in Proc.First Mobile Computing and Wireless Communication InternationalConference (MCWC), September 2006, pp. $117-122$.
[9] A. Bachir, D. Barthel, M. Heusse, and A. Duda, "Microframe preamble MAC for multihop wireless sensor networks," in Proc. IEEE InternationalConference on Communications (ICC), vol. 7, June 2006, pp. 3365 3370 .

[10] S. Mahlknecht and M. Bock, "CSMA-MPS: a minimum preamble sampling MAC protocol for low power wireless sensor networks," in Proc. IEEE International Workshop on Factory Communication Systems, September 2004, pp. $73-80$.

[11] M. Buettner, G. V. Yee, E. Anderson, and R. Han, "XMAC: a short preamble MAC protocol for duty-cycled wireless sensor networks," in Proc. SenSys, 2006, pp. 307-320.

[12] X. Shi and G. Stromberg, "SyncWUF: An ultra-lowpower MAC protocol for wireless sensor networks," IEEE Trans. Mobile Computing, vol. 6, no. 1, pp. 115 125, January 2007.

[13] Y. Sun, O. Gurewitz, and D. B. Johnson, "RI-MAC: A receiver-initiated asynchronous duty cycle MAC protocol for dynamic traffic loads in wireless sensor networks," in Proc. SenSys, 2008, pp. 1-14.

[14] P. Huang, C. Wang, L. Xiao, and H. Chen, "RC-MAC: A receiver-centric medium access control protocol for wireless sensor networks," in Proc. IWQoS, 2010, pp. 19. 